\title{
Results from a Community-Based Smoking Cessation Treatment Program for LGBT Smokers
}

\author{
Alicia K. Matthews, ${ }^{1}$ Chien-Ching Li, ${ }^{1,2}$ Lisa M. Kuhns, ${ }^{3}$ \\ Timothy B. Tasker, ${ }^{4}$ and John A. Cesario ${ }^{1}$ \\ ${ }^{1}$ University of Illinois at Chicago, College of Nursing, 845 South Damen Avenue, Chicago, IL 60612, USA \\ ${ }^{2}$ Rush University Medical Center, College of Health Sciences, Department of Health Systems Management, \\ 1635 West Congress Parkway, Chicago, IL 60612, USA \\ ${ }^{3}$ Ann \& Robert H. Lurie Children's Hospital of Chicago \& Northwestern University Feinberg School of Medicine, 225 East Chicago \\ Avenue, Chicago, IL 60611, USA \\ ${ }^{4}$ University of Illinois at Chicago, Department of Psychology, 1007 West Harrison Street, Chicago, IL 60607, USA \\ Correspondence should be addressed to Alicia K. Matthews; aliciak@uic.edu
}

Received 16 October 2012; Revised 29 April 2013; Accepted 4 May 2013

Academic Editor: Edward Trapido

Copyright (C) 2013 Alicia K. Matthews et al. This is an open access article distributed under the Creative Commons Attribution License, which permits unrestricted use, distribution, and reproduction in any medium, provided the original work is properly cited.

\begin{abstract}
Introduction. Little is known about lesbian, gay, bisexual, and transgender (LGBT) people's response to smoking cessation interventions. This descriptive study examined the benefits of a community-based, culturally tailored smoking cessation treatment program for LGBT smokers. Methods. A total of $N=198$ LGBT individuals recruited from clinical practice and community outreach participated in group-based treatment. Sessions were based on the American Lung Association's "Freedom from Smoking Program" (ALA-FFS) and were tailored to LGBT smokers' needs. Seven-day smoking point prevalence abstinence served as the primary outcome. Results. Participants $(M$ age $=40.5)$ were mostly White $(70.4 \%)$ and male $(60.5 \%)$ and had at least a college degree (58.4\%). Forty-four percent scored in the moderate range on the Fagerström Test for Nicotine Dependence pretreatment, and $42.4 \%$ completed treatment ( $\geq 75 \%$ sessions). Higher educational attainment and use of nicotine replacement therapy (NRT) were associated with treatment completion. Self-reported quit rates were $32.3 \%$ at posttreatment assessment. Treatment attendance $(\mathrm{OR}=2.45)$, use of NRT $(\mathrm{OR}=4.24)$, and lower nicotine dependency $(\mathrm{OR}=0.73)$ were positively associated with quitting smoking. Conclusions. Results suggest the benefits of offering LGBT smokers culturally tailored smoking cessation treatments. Future research could improve outcomes by encouraging treatment attendance and promoting NRT uptake.
\end{abstract}

\section{Introduction}

Members of the lesbian, gay, bisexual, and transgender (LGBT) communities have been identified as a population group at elevated risk for smoking-related health disparities $[1,2]$. For example, results from national samples of LGBT persons consistently demonstrate disparate rates of tobacco use, with prevalence rates of smoking roughly twice those of heterosexuals [2-5]. A recent study found that tobacco use contributes to the greater than expected rates of acute respiratory illnesses among LGBT young adults compared to a heterosexual comparison group [1]. Furthermore, LGBT persons have higher than expected prevalence of risk factors (e.g., heavy drinking) for diseases associated with or exacerbated by smoking (e.g., heart disease, HIV infection, and lung cancer) [6-8]. As such, tobacco prevention and control have emerged as a significant priority for researchers, policy makers, and national organizations focused on LGBT concerns. Yet, best practices for smoking cessation among LGBT populations are glaringly absent because of the lack of empirical research $[9,10]$. Testing innovations developed by community organizations, local health departments, and others outside of academic centers (i.e., practice-based research) can help address such gaps $[11,12]$. 
In the general population the majority of current smokers $(68.8 \%)$ report wanting to quit, and more than half of smokers $(52.4 \%)$ have made a quit attempt in the previous year [13]. Similarly, members of the LGBT communities are knowledgeable about the risks associated with smoking and interested in quitting smoking. For example, awareness of the health risks associated with smoking and acceptance of tobacco control activities was high in a recent study of smoking-related knowledge, attitudes, and behaviors of LGBT persons [14]. In addition, high levels of awareness of smoking prevention campaigns, stated intentions to quit smoking, and reports of recent quit attempts have recently been observed among LGBT samples $[15,16]$. With regard to treatment seeking, data collected from a large $(N=1633)$ convenience sample of current LGBT smokers suggested that the majority $(75 \%)$ were willing to talk with their providers about access to smoking cessation treatments [17]. About one-quarter of those respondents indicated their intention to use nicotine replacement therapy (NRT) during their next quit attempt [17], a rate consistent with the general population of smokers [18]. Taken together, these findings suggest both a receptivity to and an opportunity for further engaging LGBT smokers in smoking cessation efforts.

Increasing the availability of evidence-based treatments to LGBT smokers is central to reducing the high rates of tobacco use in this group [19]. Although effective smoking cessation treatments are now widely available [9], the majority of these treatments were designed as interventions for the general population and not culturally tailored to the specific needs of any particular subgroup. A large body of evidence suggests that psychosocial and cultural factors related to smoking cessation are differentially distributed among subgroups and that addressing these differences may improve treatment outcomes [9]. Indeed, a survey conducted with gay/bisexual male smokers revealed a stronger preference for smoking cessation programs tailored to gay men over generic programs [20]. Culturally tailored interventions have been shown to be effective in improving outcomes for a range of health promotion interventions [21, 22]. Examples of tailored smoking cessation interventions include programs for people with chronic illnesses such as cancer [23]; racial and ethnic minority youth [24]; and members of Hispanic [25], African American [26], and Indigenous populations [27]. As members of a highly stigmatized minority group, LGBT persons experience unique and formidable barriers to smoking cessation including stress associated with discrimination, internalized homophobia, and limited access to culturally competent health care [28]. To address these unique concerns, efforts have been made to develop culturally competent and tailored smoking cessation treatment materials that address the cultural and psychosocial needs of LGBT smokers [29-32].

Despite the availability of culturally specific smoking cessation materials, little research exists on outcomes for LGBT smokers based on either tailored or nontailored treatment programs $[2,9,33]$. One example is provided by Harding and colleagues [34] who conducted a pilot smokingcessation intervention that was culturally tailored for gay men in the United Kingdom $(N=69)$. Their intervention consisted of four weekly two-hour sessions together with NRT. Outcomes were promising with biochemically verified quit rates one month after treatment at $48 \%$. Walls and Wisneski [35] reported on a preliminary evaluation of the acceptability of a set of community-based smoking cessation classes, "The Last Drag," that were culturally tailored to the sociocultural needs of LGBT smokers $(N=44)$. The smoking cessation classes were based on a culturally adapted version of the American Lung Association's Freedom from Smoking (ALA-FFS) curriculum [36] and included seven group-based counseling and peer support sessions. Of the 44 individuals who participated, 36 were in attendance in the final class, and $89 \%$ of those self-reported being quit. Ratings of the cultural appropriateness of the program were also strong. In a larger scale evaluation of "The Last Drag" program, Eliason and colleagues [19] reported results from all participants who completed one or more sessions from August 2005 to January $2010(N=233)$. Self-reported quit rates for the sample were $53 \%$ at one month and $36 \%$ at 3 and 6 months, respectively. Finally, a recent study examined smoking cessation outcomes based on sexual orientation following a nontailored smoking cessation intervention [37]. A total of 54 sexual minority men (SMM) and 243 heterosexual male smokers received 8 weeks of treatment that included nicotine patch, Bupropion, and individual behavioral counseling sessions. Rates of quitting among SMM were nearly identical to those of heterosexual males (week 8; SMM = 59\% versus heterosexual $=57 \%$ ). The results from the few existing smoking cessation treatment programs involving LGBT smokers are promising; however, additional research is needed to establish culturally appropriate and evidence-based treatment strategies. In the absence of extensive research-based evidence, data available from existing smoking cessation treatments offered as part of routine clinical services can provide important information about smoking cessation treatments in "real world settings" and can guide future research and provide models for other community-based treatment centers.

\section{Specific Aims}

The purpose of this study was to evaluate the treatment outcomes associated with a culturally tailored smoking cessation treatment program for LGBT smokers that were offered as part of clinical practice in a community-based health center. We sought to answer the following research questions: (1) What were the participation rates of LGBT smokers taking part in a community-based smoking cessation treatment program? (2) Was there evidence that the culturally tailored programs were effective for reducing smoking behaviors? (3) What participant characteristics (demographic, smoking, and treatment attendance) were associated with better treatment outcomes?

\section{Methods}

3.1. Sample. Data for this study were obtained from a series of smoking cessation treatment programs tailored to LGBT smokers and offered at Howard Brown Health 
Center (HBHC) from 2002 to 2006. The original program, "Call It Quits," was developed as part of a research grant awarded to HBHC. After completion of the research grant, additional funding was obtained to offer smoking cessation treatments as part of clinical services at HBHC. A total of three separate programs were offered including "Call It Quits" (CIQ, 2002, a total of 105 smokers participated in 15 cessation groups), "Bitch To Quit" (BTQ, 2005-2006, a total of 33 smokers participated in 5 cessation groups), and "Put It Out" (PIO, 2006-2008, a total of 60 smokers participated in 10 cessation groups). Each of these clinical programs were based on the core CIQ curriculum; however, some variations in approaches occurred, including changes in program names, assessment measures, the inclusion of a peer support "buddy," or the number of treatment sessions offered.

The smoking cessation classes were advertised using a range of strategies including provider referrals and information posted in HBHC's medical clinic and through postings and materials (e.g., posters, flyers, and matchbooks) in community venues. When possible, additional funding was obtained to do specific outreach and advertising to subpopulations of the LGBT communities based on race/ethnicity, gender, or gender identity. These groups followed the same treatment procedures as the standard groups but were held in locations specifically serving the needs of members from these communities. In general, eligibility criteria for the smoking cessation treatment programs included (1) selfidentified as LGBT, (2) age 18-65, and (3) current cigarette smoker (any level of smoking). Depending on when smoking cessation services were received, treatment was either free of charge or provided on a sliding scale fee schedule according to income. No interested smokers were denied treatment because of inability to pay. Data from each of the treatment groups were combined for statistical analyses. The Institutional Review Board of HBHC has approved the secondary analysis of programmatic data.

3.1.1. Treatment Approach. All treatment programs were developed based on the well-established ALA-FFS, a groupbased and cost-effective group smoking cessation treatment $[36,38]$. The underlying premise of the ALA-FFS cognitive/behavioral program is that smoking is a learned habit. The program focuses on how to make behavioral changes and to quit smoking in the context of a group environment. The ALA-FFS program was selected because it incorporates many of the best practices identified by the Treatment Practice Guidelines [39] including skill building and preparation for quitting, intratreatment social support, and optimal treatment dosing (i.e., $4-7$ sessions).

Because the ALA-FFS program was designed for the mainstream USA population, the program was adapted for the LGBT community. The culturally tailored smoking cessation program maintained the core cognitive and behavioral approaches and smoking education content used in the standard ALA-FFS program. The goal of the tailoring was to adapt the existing ALA-FFS cessation materials to the culture, norms, and beliefs of LGBT men and women and to address the unique factors associated with their smoking behaviors. First we focused on strategies to increase the level of trust and acceptability of the program. These included hosting all smoking cessation treatments at LGBT community organizations, employing LGBT-identified smoking cessation counselors, and branding recruitment and program materials with LGBT-specific images. Next we included culturally specific content into treatment materials. The culturally specific content was informed by information obtained from focus group interviews with LGBT smokers that explored barriers and facilitators to smoking cessation ( $N=9$ groups) [40], published guidelines for conducting culturally competent smoking cessation treatments [41], a panel of LGBT health experts, a review of the literature, and recommendations from existing tailored smoking cessation programs (e.g., QueerTIPs [30]). A few examples of culturally tailored elements included a discussion of health concerns for LGBT smokers (e.g., HIV/AIDS, hormone use among transgender smokers); the role of smoking in the LGBT culture; stress due to homophobia as triggers for smoking and relapse; ways to increase social support for nonsmoking; relationships between bar culture, drinking, and smoking; targeting of the LGBT communities by the tobacco industry; providing smoking statistics/rates specially for the LGBT communities; and the inclusion of themes related to community empowerment and health. The combined information was folded into the ALA-FFS curriculum to create the tailored "Call It Quits" (later renamed as "Bitch To Quit" and "Put It Out") smoking cessation program materials and the training curriculum for group facilitators.

A total of 30 separate treatment groups were offered. The majority of the smoking cessation groups were conducted at the HBHC. Additional funding was obtained to offer CIQ at three other community-based locations serving the needs of LGBT smokers from diverse ethnic and racial backgrounds. LGBT-identified professional facilitators conducted each group session. In the CIQ sessions only, LGBT exsmokers served as peer support "buddies." Smoking cessation counselors were trained through the American Lung Association of Metropolitan Chicago (ALAMC). Treatment groups were offered once weekly and ran for approximately 90 minutes each. The number of weekly treatment sessions varied somewhat across the differing treatment groups (CIQ $=8$ sessions, $\mathrm{BTQ}=8$ sessions, $\mathrm{PIO}=6$ sessions). Outcome and process measures were collected at baseline, weekly, and at the end of treatment (approximately one month after quit date).

3.1.2. Nicotine Replacement. NRT (i.e., patches) was provided free of charge as part of the PIO sessions from 2006 to 2008. Use of nicotine replacement and other pharmacotherapies during the course of treatment was measured for all treatment participants regardless of whether NRT was offered as part of treatment.

\subsection{Measures}

3.2.1. Participant Background Characteristics. Demographic characteristics measured as part of the baseline assessment 
included age, race (Asian, Black, White, other), gender (male, female, transgender), education (high school or lower, some college, college and above), employment status (full time, part time, unemployed, retired, disabled, other), annual income (\$0-10,999, \$11-20,999, \$21-30,999, \$31-49,999, \$50,000 and over), and sexual orientation (gay, lesbian, bisexual, other).

3.2.2. Smoking Behaviors and Attitudes. Nicotine dependence was measured by the Fagerström Test for Nicotine Dependence [42]. This test assesses a smoker's level of dependency on nicotine and has demonstrated validity and reliability in previous research. Scores range from 0 to 10 with higher scores suggesting more nicotine dependence. Nicotine dependence was classified into three levels based on these composite scores: low (0-3), medium (4-6), and high (7-10). Smoking frequency was asked by the following single item: "How many cigarettes/day do you smoke?" (3140 or more cigarettes, 21-30 cigarettes, 11-20 cigarettes, 10 or less cigarettes). Attitudes towards quitting were measured at baseline using four individual questions developed to measure cognitive factors associated with quitting behavior: (1) "How much do you want to quit smoking?" (definitely not, probably not, not sure, probably yes, definitely yes); (2) "How important is it for you to quit smoking?" (not at all, a little, moderately, very, extremely); (3) "Do you intend to quit smoking?" (definitely not, probably not, not sure, probably yes, definitely yes); and (4) "How confident are you in your ability to quit smoking completely?" (not at all, a little, moderately, very, extremely). These items were all worded in the same direction such that higher scores on each corresponded to more agreement with the question.

3.2.3. Treatment Outcomes. The primary outcome measured was self-reported seven-day point prevalence abstinence measured at completion of the smoking cessation intervention sessions. Individuals who had missing data for quitsmoking status were treated as nonquit based on an intention to-treat (ITT) approach to data analysis. Session attendance, a measure of treatment dose, was also measured as an important treatment outcome. Given that the number of treatment sessions differed somewhat between treatment programs ( $\mathrm{CIQ}=8$ sessions, $\mathrm{BTQ}=8$ sessions, and $\mathrm{PIO}=6$ sessions $)$, treatment completion was operationally defined as attending $75 \%$ or more of all available treatment sessions. Finally, all participants were asked whether they used any kind of stopsmoking medications during treatment, including nicotine replacement (e.g., patch, gum) or medications (e.g., Chantix, Bupropion).

3.3. Data Analysis. Data were available from $N=198$ LGBT smokers who completed at least one session of the culturally tailored smoking cessation programs. Descriptive statistics were computed for all variables. Comparisons of characteristics between smoking cession groups (i.e., CIQ, BTQ, and PIO) were performed using Chi-Square $\left(\chi^{2}\right)$ tests for categorical variables and Analysis of Variance (ANOVA) for continuous variables. Multivariate binary logistic regression was performed to describe associations between smoking status and demographic characteristics, smoking attitudes, smoking characteristics, and treatment factors (i.e., use of NRT and number of sessions completed). Finally, additional multivariate linear regression analyses were conducted to examine the associations between session attendance and demographic characteristics, smoking attitudes, smoking characteristics, and treatment factors. All analyses were performed in SAS version 9.2.

\section{Results}

4.1. Demographic Characteristics. The mean age of the participants was 40.48 years $(\mathrm{SD}=9.22)$. The majority of participants were White $(70.4 \%)$ and male $(60.5 \%)$ and had at least a college degree (58.4\%). As shown in Table 1, the participants in each of the three smoking cessation treatment programs differed significantly based on demographic characteristics. CIQ had the highest proportion of male $(76.7 \%)$ and unemployed people (43.3\%); BTQ had the highest proportion of non-White (39.4\%), female (93.9\%), employed people $(75.8 \%)$, and annual income more than $\$ 50,000$ (45.5\%); and PIO had the highest proportion of White people $(83.3 \%)$, with these differences reaching statistical significance. Accordingly, a set of key demographic factors was used as control variables in subsequent analyses.

4.2. Smoking Behaviors and Smoking Cession Outcomes. At pretreatment, the majority of program participants reported definitely wanting to $(62.4 \%)$ and intending to quit smoking $(57.2 \%)$, and the vast majority of all participants indicated that it was either very or extremely important to quit smoking (85.1\%). However, the proportion reporting that they were either very or extremely confident in their ability to quit smoking was comparatively lower (43.3\%). The highest proportion of participants smoked 11-20 cigarettes per day (39.9\%). Another $35.9 \%$ were light smokers (0-10 cigarettes per day) and $24.3 \%$ were heavy smokers (21+ cigarettes per day). Based on the Fagerström measure, mean nicotine dependency scores for all participants indicated moderate levels of dependency $(M=4.18, \mathrm{SD}=2.49)$. Nearly one-fifth of the sample (17.9\%) scored in the high nicotine dependence range on the Fagerström measure. Significant differences in smoking characteristics were also observed for participants in each of the three different treatment groups (see Table 1). Treatment attendance rates were modest with $42.4 \%$ of participants completing $\geq 75 \%$ of sessions. Overall $32.3 \%$ of all participants self-reported having quit at the end of treatment.

4.3. Multivariate Predictors of Treatment Outcomes. Multivariate analyses were conducted to assess the association between quit-smoking status and smoking characteristics (nicotine dependence), attitudes about quitting (want to quit, important to quit, intend to quit, confidence to quit), and treatment factors (session attendance and use of stopsmoking medications) controlling for demographic characteristics (race, gender, education, sexual orientation). Results showed that baseline levels of nicotine dependence, use of any stop-smoking medications as an adjunct to group counseling, 
TABLE 1: Demographic, attitudinal, and smoking characteristics by treatment program offered.

\begin{tabular}{|c|c|c|c|c|c|}
\hline Variable & Total $(N=198)$ & CIQ $(n=105)$ & BTQ $(n=33)$ & PIO $(n=60)$ & \\
\hline Demographic characteristics & & Mean $(\mathrm{SD}$ & cent $\%$ & & $p$ values \\
\hline Age & $40.48(9.22)$ & $41.50(8.83)$ & $38.00(8.58)$ & $40.31(9.99)$ & $\mathrm{N} / \mathrm{S}$ \\
\hline Race & & & & & $<0.05$ \\
\hline White & 70.43 & 65.59 & 60.61 & 83.33 & \\
\hline Non-White & 29.57 & 34.41 & 39.39 & 16.67 & \\
\hline Gender & & & & & $<0.05$ \\
\hline Male & 60.51 & 76.70 & 0.00 & 66.10 & \\
\hline Female & 35.38 & 17.48 & 93.94 & 33.90 & \\
\hline Transgender & 4.10 & 5.83 & 6.06 & 0.00 & \\
\hline Education & & & & & $\mathrm{N} / \mathrm{S}$ \\
\hline High school or lower & 11.68 & 15.38 & 6.06 & 8.33 & \\
\hline Some college & 29.95 & 31.73 & 27.27 & 28.33 & \\
\hline College or above & 58.38 & 52.88 & 66.67 & 63.33 & \\
\hline Employment status & & & & & $<0.05$ \\
\hline Full time & 59.18 & 49.04 & 75.76 & 67.80 & \\
\hline Part time & 6.12 & 7.69 & 0.00 & 6.78 & \\
\hline Unemployed & 34.69 & 43.27 & 24.24 & 25.42 & \\
\hline Annual income & & & & & $<0.05$ \\
\hline$\$ 0-10,999$ & 24.35 & 33.01 & 9.09 & 17.54 & \\
\hline$\$ 11-20,999$ & 12.44 & 15.53 & 3.03 & 12.28 & \\
\hline$\$ 21-30,999$ & 12.95 & 14.56 & 6.06 & 14.04 & \\
\hline$\$ 31-49,999$ & 22.28 & 21.36 & 36.36 & 15.79 & \\
\hline$\$ 50,000$ and over & 27.98 & 15.53 & 45.45 & 40.35 & \\
\hline Sexual orientation & & & & & $\mathrm{N} / \mathrm{S}$ \\
\hline Homosexual & 75.51 & 76.92 & 71.88 & 75.00 & \\
\hline Bisexual & 10.71 & 8.65 & 12.50 & 13.33 & \\
\hline Others & 13.78 & 14.42 & 15.63 & 11.67 & \\
\hline Smoking characteristics & & & & & \\
\hline How much do you want to quit? & & & & & $\mathrm{N} / \mathrm{S}$ \\
\hline Definitely not & 1.55 & 1.90 & 0.00 & 1.79 & \\
\hline Probably not & 0.00 & 0.00 & 0.00 & 0.00 & \\
\hline Not sure & 3.61 & 3.81 & 6.06 & 1.79 & \\
\hline Probably yes & 32.47 & 33.33 & 30.30 & 32.14 & \\
\hline Definitely yes & 62.37 & 60.95 & 63.64 & 64.29 & \\
\hline How important to quit? & $4.34(0.77)$ & $4.23(0.78)$ & $4.30(0.81)$ & $4.57(0.68)$ & $<0.05$ \\
\hline Not at all & 0.00 & 0.00 & 0.00 & 0.00 & \\
\hline A little & 1.55 & 0.95 & 6.06 & 0.00 & \\
\hline Moderately & 13.40 & 18.10 & 3.03 & 10.71 & \\
\hline Very & 34.54 & 38.10 & 45.45 & 21.43 & \\
\hline Extremely & 50.52 & 42.86 & 45.45 & 67.86 & \\
\hline Do you intend to quit? & & & & & $<0.05$ \\
\hline Definitely not & 0.00 & 0.00 & 0.00 & 0.00 & \\
\hline Probably not & 0.00 & 0.00 & 0.00 & 0.00 & \\
\hline Not sure & 9.79 & 12.38 & 9.09 & 5.36 & \\
\hline Probably yes & 32.99 & 40.95 & 18.18 & 26.79 & \\
\hline Definitely yes & 57.22 & 46.67 & 72.73 & 67.86 & \\
\hline How confident to quit? & $3.36(0.99)$ & $3.33(0.99)$ & $3.55(0.90)$ & $3.30(1.06)$ & $\mathrm{N} / \mathrm{S}$ \\
\hline Not at all & 2.58 & 1.90 & 3.03 & 3.57 & \\
\hline A little & 15.98 & 17.14 & 9.09 & 17.86 & \\
\hline Moderately & 38.14 & 40.95 & 27.27 & 39.29 & \\
\hline Very & 29.38 & 25.71 & 51.52 & 23.21 & \\
\hline Extremely & 13.92 & 14.29 & 9.09 & 16.07 & \\
\hline
\end{tabular}


TABle 1: Continued.

\begin{tabular}{|c|c|c|c|c|c|}
\hline Variable & Total $(N=198)$ & CIQ $(n=105)$ & BTQ $(n=33)$ & $\mathrm{PIO}(n=60)$ & \\
\hline Demographic characteristics & \multicolumn{4}{|c|}{ Mean (SD)/percent \% } & $p$ values \\
\hline Nicotine dependence & $4.18(2.49)$ & $4.64(2.45)$ & $3.87(2.58)$ & $3.30(2.29)$ & $<0.05$ \\
\hline Low & 37.72 & 28.87 & 46.67 & 52.50 & \\
\hline Moderate & 44.31 & 50.52 & 36.67 & 35.00 & \\
\hline High & 17.96 & 20.62 & 16.67 & 12.50 & \\
\hline Nicotine replacement therapy & & & & & $<0.05$ \\
\hline Yes & 24.24 & 27.62 & 36.36 & 11.67 & \\
\hline No & 75.76 & 72.38 & 63.64 & 88.33 & \\
\hline \multicolumn{6}{|l|}{ Smoking cession outcomes } \\
\hline Number of sessions attended & $5.37(2.03)$ & $6.19(1.74)$ & $6.55(0.89)$ & $3.48(1.47)$ & $<0.05$ \\
\hline Program completing & & & & & $<0.05$ \\
\hline$\geq 75 \%$ & 42.42 & 52.38 & 54.55 & 31.67 & \\
\hline$<75 \%$ & 57.58 & 47.62 & 45.45 & 68.33 & \\
\hline Quit rates (ITT at 1 month) & & & & & $\mathrm{N} / \mathrm{S}$ \\
\hline Yes & 32.32 & 39.05 & 27.27 & 23.33 & \\
\hline No & 67.68 & 60.95 & 72.73 & 76.67 & \\
\hline
\end{tabular}

CIQ: Call It Quits; BTQ: Bitch To Quit; PIO: Put It Out; SD: standard deviation; N/S: not significant; ITT: intent-to-treat analyses.

and number of sessions attended were significantly associated with quit-smoking status. Any use of nicotine replacement $(\mathrm{OR}=4.24,95 \% \mathrm{CI}=1.23-14.50)$ and increased number of sessions attended $(\mathrm{OR}=2.45,95 \% \mathrm{CI}=1.45-4.15)$ were associated with higher odds of quitting smoking, respectively. However, increased nicotine dependence scores were associated with lower odds of quitting smoking (OR $=0.73,95 \% \mathrm{CI}$ $=0.57-0.94$ ) (see Table 2).

Multivariate analyses were also performed to examine the associations between session attendance rates and smoking characteristics (nicotine dependence), attitudes about quitting (want to quit, important to quit, intend to quit, confidence to quit), and treatment factors (nicotine replacement use) while controlling for each demographic characteristic (race, gender, education, sexual orientation). After introducing all demographic and smoking characteristics in the multivariate model, education and nicotine replacement use still significantly associated with session attendance. Participants with some college education or less had lower session attendance rates compared to those with a college degree or more education $(\beta=-0.10, p<0.05)$. People who had ever used nicotine replacement had higher session attendance rates than those who had not used it $(\beta=0.10$, $p<0.05$ ) (see Table 3).

\section{Discussion}

Scant research exists on effective smoking cessation treatments for LGBT smokers. Evaluation of smoking cessation treatments offered as part of clinical or behavioral health services provides opportunities to answer important questions about LGBT smoking cessation and can help establish the evidence base for treating tobacco addiction in LGBT smokers. The purpose of this paper was to address a significant gap in the literature on tobacco cessation by reporting on the outcomes associated with culturally tailored treatment programs for LGBT smokers. Study results suggest
TABLe 2: Multivariate analysis of factors associated with quitsmoking status $(N=121)$.

\begin{tabular}{lccc}
\hline Variable & Odds ratio & $95 \%$ CI & $p$ values \\
\hline Nicotine dependence & 0.73 & $0.57-0.94$ & 0.01 \\
Nicotine replacement therapy & 4.24 & $1.24-14.50$ & 0.02 \\
Number of sessions attended & 2.45 & $1.45-4.15$ & $<0.001$ \\
\hline
\end{tabular}

Race, gender, education, sexual orientation, attitudes about quitting smoking (want to quit, important to quit, intend to quit, and confidence to quit), and treatment program were adjusted for in the model.

that culturally specific smoking cessation treatments offered in a community setting are beneficial for reducing smoking among LGBT smokers. Contrary to data suggesting low awareness and engagement of LGBT smokers with formal treatment programs [16], we demonstrated the ability to enroll and retain LGBT smokers into a community-based and intensive group smoking cessation program. Further, quit rates were on average consistent with outcomes associated with general population results from the ALA-FFS program [36]. These promising findings warrant future research to replicate results and to examine differences in smoking cessation outcomes from the tailored and nontailored ALAFFS programs.

5.1. Quit Rates and Factors Associated with Quitting. Overall quit rates were in the expected range for community-based smoking cessation treatments and ranged from 23.3 to $39.1 \%$ at the end of treatment. These intent-to-treat, quit rates at end of treatment and followup are comparable to those observed in mainly Caucasian smokers engaged in similar treatment (i.e., with nicotine patch and intensive counseling) [9]. However, the same contextual factors observed in LGBT communities that hinder smoking cessation (e.g., stress associated with discrimination, fewer deterrents to smoking, and limited access to culturally competent health care) [28] 
TABLE 3: Multivariate analysis of factors associated with session attendance rates $(N=121)$.

\begin{tabular}{lccc}
\hline Variable & $\begin{array}{c}\text { Beta } \\
\text { coefficient }\end{array}$ & S.E. & $p$ values \\
\hline $\begin{array}{l}\text { Education (reference: } \\
\text { college or above) } \\
\quad \text { Some college }\end{array}$ & -0.10 & 0.04 & 0.03 \\
$\begin{array}{l}\text { Nicotine replacement } \\
\text { therapy (reference: no) } \\
\quad \text { Yes }\end{array}$ & 0.10 & 0.04 & 0.01 \\
\hline
\end{tabular}

Race, gender, sexual orientation, attitudes about quitting smoking (want to quit, important to quit, intend to quit, and confidence to quit), nicotine dependence, and treatment program were adjusted for in the model. Only significant factors are shown in the table.

may also serve as formidable barriers to long-term cessation and should be systematically addressed as part of intensive relapse prevention treatment. As such, additional research is needed to examine maintenance of quit rates over time.

In the general population, quit rates following smoking cessation treatment vary substantially based on demographic characteristics such as race/ethnicity. However, in multivariate analyses, demographic characteristics were not statistically significant predictors of the quit status of program participants. Instead, smoking- and treatment-related variables had direct effects on quit outcomes. The likelihood of being a nonsmoker was positively associated with use of stop-smoking medications and session attendance rates such that the odds of quitting were more than doubled with the use of any medications and completion of more treatment sessions. It should be noted that, in general, the use of stop-smoking medications was relatively low across program participants (24.2\%). In general, nicotine dependency scores were in the moderate range and were associated with quit status such that those smokers with higher levels of nicotine dependency had lower odds of being quit after treatment. The relationship between nicotine dependence and lower quit rates suggests the need for increasing awareness and uptake of pharmacological approaches as key adjuncts to stop-smoking counseling programs.

Treatment completion rates were modest at $42.4 \%$ (range $31.7 \%-54.6 \%)$. After introducing all demographic and smoking characteristics in the multivariate model, education level and nicotine replacement use were significantly and positively associated with session attendance rates. Given the association between treatment attendance and quit outcomes, future initiatives should focus on strategies increasing adherence to treatment.

5.2. Improving LGBT Smoking Cessation Interventions. A strength of the smoking cessation treatments offered at $\mathrm{HBHC}$ was the adaption of an existing and evidence-based program. This approach was consistent with calls to increase access to treatments for priority populations by disseminating effective behavioral interventions [11]. Other strategies to increase the impact and cultural competency of smoking cessation treatments have been well described [41] and minimally include the following: (1) conduct the treatment programs in settings that are considered safe to community members; (2) treatments should be administered by facilitators with expertise in smoking cessation and cultural competency in working with the LGBT communities; (3) distribute appropriate, inclusive, and creative promotional materials through community-based outlets; (4) provide cultural competency training to all non-LGBT staff who interact with participants; and (5) modify the curricula to include LGBT-specific and other culturally relevant information for participants. In addition, our findings suggest that the core cognitive and behavioral components of the ALA-FFS curriculum are beneficial for reducing smoking behaviors. Consistent with treatment and practice guidelines and our findings, it is important to include stop-smoking medications as a standard part of treatment. That being said, adherence to NRT was low among participants even when it was offered free of charge. As such, additional emphasis should be placed on identifying and addressing barriers to uptake of NRT as a part of standard treatment.

In addition, focus groups were used to tailor these treatment programs to the needs of LGBT smokers. Several years have passed since this qualitative research was conducted and the findings may no longer be applicable to the current smokers based on a number of factors including identity factors (e.g., queer, gender nonconforming), smoking behaviors (e.g., menthol, light smokers, use of new tobacco products), barriers and facilitators to smoking cessation, and types of images and messaging that are appropriate. Consequently, additional formative research may be needed to revise and refine materials to address the needs of current LGBT smokers.

5.3. Strengths and Limitations. The tailored curriculum retained the essential effective components of the ALAFFS program, only deviating from the standard intervention in minimal ways. Further, facilitators were trained by the ALAMC. As such, we believe we were able to maintain the integrity of the program and that the adapted intervention was largely equivalent to the standard ALA-FFS program. Additional strengths of the study included culturally tailoring an existing and effective smoking cessation treatment program for LGBT smokers, measurement of demographic and smoking variables that may impact treatment outcomes, and posttreatment follow-up of treatment participants. However, the results should be considered in light of the study limitations. For example, the majority of these quit rates were not biochemically confirmed by objective measures and there was no comparison control condition. As such, these initial promising findings need replication and extension using more robust methodology. Additionally, we did not collect any culturally specific measures (e.g., minority stress) that may have provided additional insight into factors that may influence within-group differences in smoking behavior. Although basic demographic and smoking variables were measured as part of the treatment programs, a full battery of smoking questions was not included. Finally, no data were available on longer-term quit rates associated with the program. 


\section{Conclusions}

Study findings demonstrate the feasibility and benefits of providing smoking cessation treatments for LGBT smokers as part of clinical services. Future studies should build on the success of this clinical program of smoking cessation treatment by systematically evaluating the benefits of a culturally tailored intervention for improving smoking cessation outcomes in LGBT smokers. A research program should address the limitations of our clinical program by (1) improving the internal validity of the intervention (randomization, inclusion of a control condition, and adequate power); (2) incorporating smoking cessation intervention "best practices" (objective verification of smoking status, NRT, and long-term followup); and (3) systematically tailoring the ALA-FFS intervention modules (focus group research to guide development of peripheral, evidential, linguistic, sociocultural, and constituent tailoring strategies). A research study such as this would allow for a more rigorous test of the added benefits of culturally tailoring the ALA-FFS program for LGBT smokers.

\section{Acknowledgments}

This research was supported by the American Legacy Foundation Grant no. 2667 awarded to Howard Brown Health Center and the Eunice Kennedy Shriver National Institute of Child Health and Human Development R21HD051178 awarded to Judith Bradford. In addition, the Research Open Access Publishing Fund at the University of Illinois at Chicago provided financial support for the open access publishing fee for this paper. The content is solely the responsibility of the authors and does not necessarily represent the official views of the NICHD or the National Institutes of Health. The authors would also like to acknowledge Drs. David McKirnan, Simone Koelinger, and Scott Cook for their important role in the development and evaluation of the initial "Call It Quits" program, Michael Mahoney on his assistance with earlier statistical analyses, and Joseph Lee for his critical review and insights.

\section{References}

[1] J. Blosnich, T. Jarrett, and K. Horn, "Disparities in smoking and acute respiratory illnesses among sexual minority young adults," Lung, vol. 188, no. 5, pp. 401-407, 2010.

[2] J. G. L. Lee, G. K. Griffin, and C. L. Melvin, "Tobacco use among sexual minorities in the USA, 1987 to May 2007: a systematic review," Tobacco Control, vol. 18, no. 4, pp. 275-282, 2009.

[3] H. Tang, G. L. Greenwood, D. W. Cowling, J. C. Lloyd, A. G. Roeseler, and D. G. Bal, "Cigarette smoking among lesbians, gays, and bisexuals: how serious a problem? (United States)," Cancer Causes and Control, vol. 15, no. 8, pp. 797-803, 2004.

[4] E. P. Gruskin, G. L. Greenwood, M. Matevia, L. M. Pollack, and L. L. Bye, "Disparities in smoking between the lesbian, gay, and bisexual population and the general population in California," The American Journal of Public Health, vol. 97, no. 8, pp. 14961502, 2007.

[5] B. A. King, S. R. Dube, and M. A. Tynan, "Current tobacco use among adults in the United States: findings from the National
Adult Tobacco Survey," The American Journal of Public Health, vol. 102, no. 11, pp. e93-e100, 2012.

[6] U. Boehmer, D. J. Bowen, and G. R. Bauer, "Overweight and obesity in sexual-minority women: evidence from populationbased data," The American Journal of Public Health, vol. 97, no. 6, pp. 1134-1140, 2007.

[7] P. Case, B. Austin, D. J. Hunter et al., "Sexual orientation, health risk factors, and physical functioning in the nurses' health study II," Journal of Women's Health, vol. 13, no. 9, pp. 1033-1047, 2004.

[8] S. D. Cochran, V. M. Mays, D. Bowen et al., "Cancer-related risk indicators and preventive screening behaviors among lesbians and bisexual women," The American Journal of Public Health, vol. 91, no. 4, pp. 591-597, 2001.

[9] M. C. Fiore and C. R. Jaén, "A clinical blueprint to accelerate the elimination of tobacco use," Journal of the American Medical Association, vol. 299, no. 17, pp. 2083-2085, 2008.

[10] Institute of Medicine, The Health of Lesbian, Gay, Bisexual, and Transgender People: Building a Foundation for Better Understanding, The National Academies Press, Washington, DC, USA, 2011.

[11] L. W. Green, "Making research relevant: if it is an evidencebased practice, where's the practice-based evidence?" Family Practice, vol. 25, supplement 1, pp. i20-i24, 2008.

[12] R. L. Miller and M. Shinn, "Learning from communities: overcoming difficulties in dissemination of prevention and promotion efforts," The American Journal of Community Psychology, vol. 35, no. 3-4, pp. 169-183, 2005.

[13] Centers for Disease Control and Prevention, "Quitting smoking among adults-United States, 2001-2010," Morbidity and Mortality Weekly Report, vol. 60, no. 44, pp. 1513-1519, 2011.

[14] B. A. Pizacani, K. Rohde, C. Bushore et al., "Smoking-related knowledge, attitudes and behaviors in the lesbian, gay and bisexual community: a population-based study from the U.S. Pacific Northwest," Preventive Medicine, vol. 48, no. 6, pp. 555561, 2009.

[15] J. E. Burkhalter, C. M. Springer, R. Chhabra, J. S. Ostroff, and B. D. Rapkin, "Tobacco use and readiness to quit smoking in lowincome HIV-infected persons," Nicotine and Tobacco Research, vol. 7, no. 4, pp. 511-522, 2005.

[16] A. K. Matthews, A. Hotton, S. Dubois, D. Fingerhut, and L. M. Kuhns, "Demographic, psychosocial, and contextual correlates of tobacco use in sexual minority women," Research in Nursing and Health, vol. 34, no. 2, pp. 141-152, 2011.

[17] A. H. Levinson, N. Hood, R. Mahajan, and R. Russ, "Smoking cessation treatment preferences, intentions, and behaviors among a large sample of Colorado gay, lesbian, bisexual, and transgendered smokers," Nicotine and Tobacco Research, vol. 14, no. 8, pp. 910-918, 2012.

[18] K. K. Ryan, E. Garrett-Mayer, A. J. Alberg, K. B. Cartmell, and M. J. Carpenter, "Predictors of cessation pharmacotherapy use among Black and non-Hispanic White smokers," Nicotine and Tobacco Research, vol. 13, no. 8, pp. 646-652, 2011.

[19] M. J. Eliason, S. L. Dibble, R. Gordon, and G. B. Soliz, "The Last Drag: an evaluation of an LGBT-specific smoking intervention," Journal of Homosexuality, vol. 59, no. 6, pp. 864-878, 2012.

[20] D. Schwappach, "Smoking behavior, intention to quit, and preferences toward cessation programs among gay men in Zurich, Switzerland," Nicotine and Tobacco Research, vol. 10, no. 12, pp. 1783-1787, 2008.

[21] M. W. Kreuter and C. S. Skinner, “Tailoring: what's in a name?" Health Education Research, vol. 15, no. 1, pp. 1-4, 2000. 
[22] M. W. Kreuter, V. J. Strecher, and B. Glassman, "One size does not fit all: the case for tailoring print materials," Annals of Behavioral Medicine, vol. 21, no. 4, pp. 276-283, 1999.

[23] M. B. Steinberg, J. Randall, S. Greenhaus, A. C. Schmelzer, D. L. Richardson, and J. L. Carson, "Tobacco dependence treatment for hospitalized smokers: a randomized, controlled, pilot trial using varenicline," Addictive Behaviors, vol. 36, no. 12, pp. 11271132, 2011.

[24] G. Kong, N. Singh, and S. Krishnan-Sarin, "A review of culturally targeted/tailored tobacco prevention and cessation interventions for minority adolescents," Nicotine an Tobacco Research, 2012.

[25] M. S. Webb, D. Rodríguez-Esquivel, and E. A. Baker, "Smoking cessation interventions among Hispanics in the United States: a systematic review and mini meta-analysis," The American Journal of Health Promotion, vol. 25, no. 2, pp. 109-118, 2010.

[26] A. K. Matthews, L. Sánchez-Johnsen, and A. King, "Development of a culturally targeted smoking cessation intervention for african american smokers," Journal of Community Health, vol. 34, no. 6, pp. 480-492, 2009.

[27] K. V. Carson, M. P. Brinn, M. Peters, A. Veale, A. J. Esterman, and B. J. Smith, "Interventions for smoking cessation in Indigenous populations," Cochrane Database of Systematic Reviews, no. 1, Article ID CD009046, 2012.

[28] S. D. Cochran and V. M. Mays, "Estimating prevalence of mental and substance using disorders among lesbians and gay men from existing national health data," in Sexual Orientation and Mental Health: Examining Identity and Development in Lesbian, Gay, and Bisexual People, A. M. Omoto and H. Kurtzmen, Eds., pp. 143-166, American Psychological Association Books, Washington, DC, USA, 2006.

[29] K. Bornstein, S. J. Israel, Skyline Media, DVN, Inc., and American Legacy Foudation, Taking the Last Drag, The Lesbian, Gay, Bisexual and Transgendered Community Center, New York, NY, USA, 2003.

[30] G. L. Greenwood and C. Hunt, QueerTIPS For LGBT Smokers, University of California, San Francisco and Progressive Research Training for Action, San Francisco, Calif, USA, 2002.

[31] Scout, A. Miele, J. B. Bradford, and D. Perry, Running an LGBT Smoking Treatment Group, The Fenway Institute, Boston, Mass, USA, 2006

[32] J. Treiber, "Developing culturally competent evaluation tools with tobacco control program practitioners," Health Promotion Practice, vol. 12, no. 5, pp. 673-680, 2011.

[33] D. M. Doolan and E. S. Froelicher, "Efficacy of smoking cessation intervention among special populations: review of the literature from 2000 to 2005," Nursing Research, vol. 55, no. 4, pp. S29-S37, 2006.

[34] R. Harding, J. Bensley, and N. Corrigan, "Targeting smoking cessation to high prevalence communities: outcomes from a pilot intervention for gay men," BMC Public Health, vol. 4, article 43, 2004.

[35] N. E. Walls and H. Wisneski, "Evaluation of smoking cessation classes for the lesbian, gay, bisexual, and transgender community," Journal of Social Service Research, vol. 37, no. 1, pp. 99-111, 2011.

[36] H. A. Lando, P. G. McGovern, F. X. Barrios, and B. D. Etringer, "Comparative evaluation of American Cancer Society and American Lung Association smoking cessation clinics," The American Journal of Public Health, vol. 80, no. 5, pp. 554-559, 1990.
[37] L. S. Covey, J. Weissman, C. LoDuca, and N. Duan, "A comparison of abstinence outcomes among gay/bisexual and heterosexual male smokers in an intensive, non-tailored smoking cessation study," Nicotine and Tobacco Research, vol. 11, no. 11, pp. 1374-1377, 2009.

[38] P. Rosenbaum and R. O'Shea, "Large-scale study of freedom from smoking clinics: factors in quitting," Public Health Reports, vol. 107, no. 2, pp. 150-155, 1992.

[39] M. C. Fiore, "A clinical practice guideline for treating tobacco use and dependence: a US public health service report," Journal of the American Medical Association, vol. 283, no. 24, pp. 32443254,2000

[40] S. Koehinger, C. Powers, B. Masini, and D. McKirnan, "Examining the effectiveness of a culturally targeted cessation program for LGBT smokers," in Proceedings of the Annual Meeting of the American Public Health Association, Philadelphia, Pa, USA, 2005.

[41] Scout, A. Miele, J. B. Bradford, and D. Perry, How to Run a Culturally Competent LGBT Smoking Treatment Group, The Fenway Institute, Boston, Mass, USA, 2007.

[42] T. F. Heatherton, L. T. Kozlowski, R. C. Frecker, and K. O. Fagerström, "The Fagerström test for nicotine dependence: a revision of the Fagerström tolerance questionnaire," British Journal of Addiction, vol. 86, no. 9, pp. 1119-1127, 1991. 


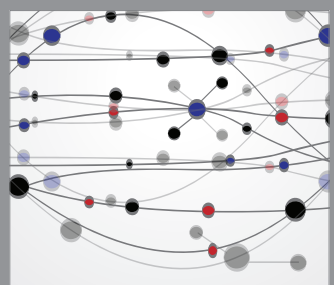

The Scientific World Journal
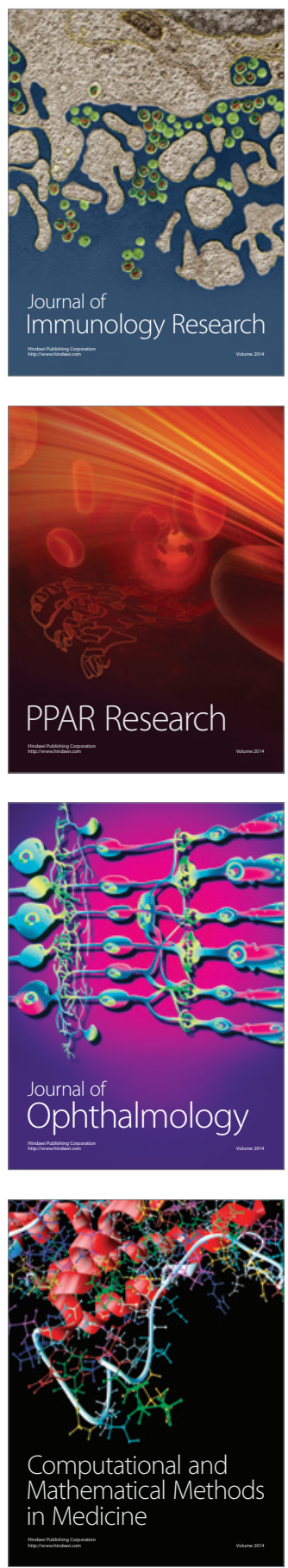

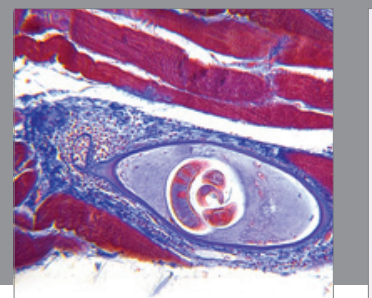

Gastroenterology

Research and Practice
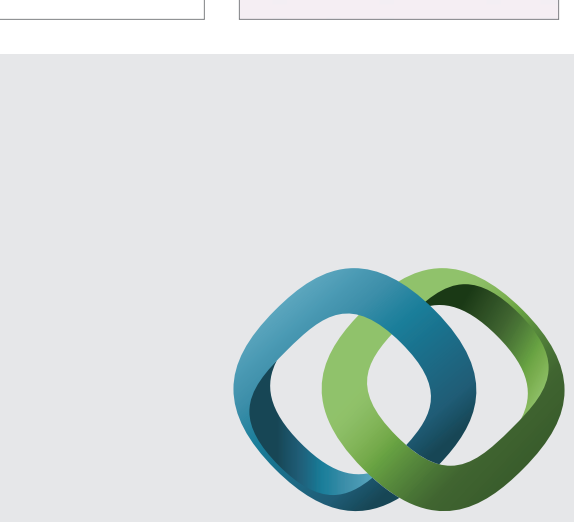

\section{Hindawi}

Submit your manuscripts at

http://www.hindawi.com
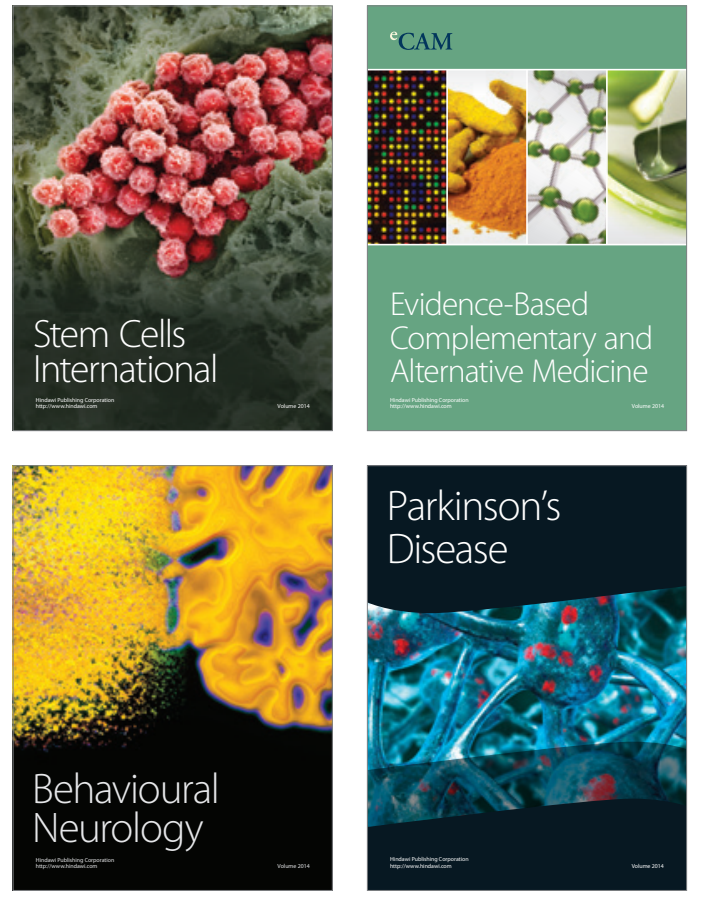
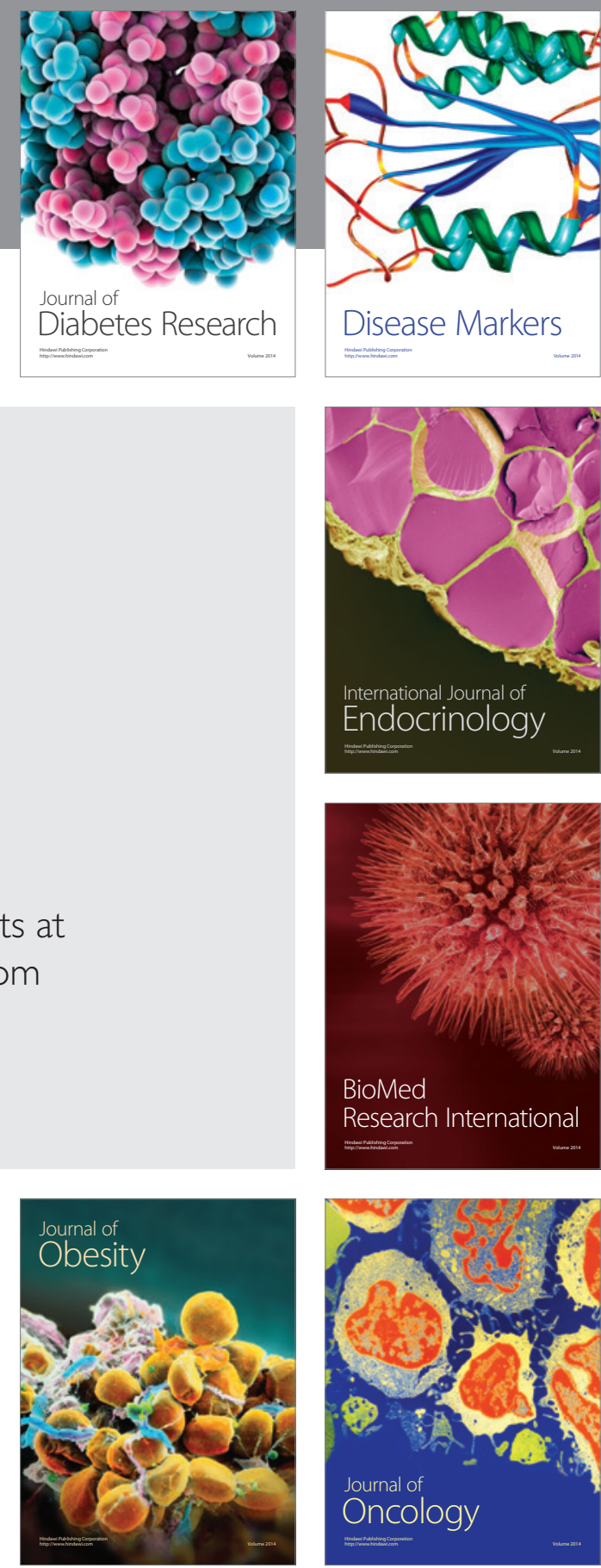

Disease Markers
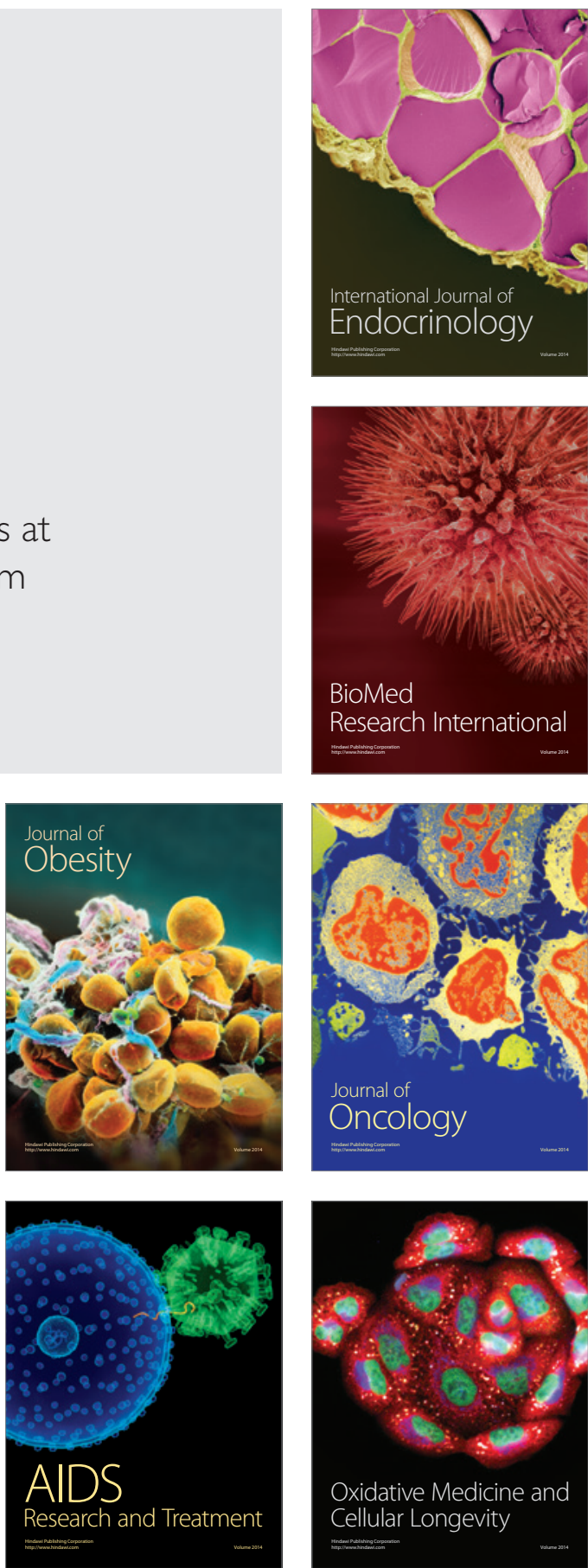